\title{
LYAPUNOV-TYPE INEQUALITIES FOR TWO CLASSES OF DIRICHLET QUASILINEAR SYSTEMS
}

\author{
Aydin TIRYAKI, DeVrim ÇAKMAK And Mustafa FAHri AKTAŞ
}

Abstract. In this paper, we establish several new Lyapunov-type inequalities for two classes of Dirichlet quasilinear systems, which almost generalize and extend all related existing results in the literature. As an application, we also obtain sharp lower bounds for the eigenvalues of corresponding systems.

Mathematics subject classification (2010): 26D10, 34A40, 34C10.

Keywords and phrases: Lyapunov-type inequalities; Quasilinear systems; $\left(p_{1}, p_{2}, \ldots, p_{n}\right)$-Laplacian; Lower bounds; Half-linear differential equation.

\section{REFERENCES}

[1] M. F. AKtaş, D. ÇaKmaK, A. TiRyaKi, A note on Tang and He's paper, Appl. Math. Comput. 218 (2012), 4867-4871.

[2] A. M. Liapunov, Probléme général de la stabilité du mouvement, Ann. Fac. Sci. Univ. Toulouse 2 (1907), 203-407.

[3] S. S. ChenG, Lyapunov inequalities for differential and difference equations, Fasc. Math. 23 (1991), $25-41$.

[4] R. C. Brown, D. B. Hinton, Opial's inequality and oscillation of 2 nd order equations, Proc. Amer. Math. Soc. 125 (1997), 1123-1129.

[5] S. S. CHENG, A discrete analogue of the inequality of Lyapunov, Hokkaido Math. J. 12 (1983), 105112.

[6] D. ÇакмАK, Lyapunov-type integral inequalities for certain higher order differential equations, Appl. Math. Comput. 216 (2010), 368-373.

[7] D. ÇAKMAK, A. TIRYAKI, On Lyapunov-type inequality for quasilinear systems, Appl. Math. Comput. 216 (2010), 3584-3591.

[8] D. ÇAKMAK, A. TIRYAKI, Lyapunov-type inequality for a class of Dirichlet quasilinear systems involving the $\left(p_{1}, p_{2}, \ldots, p_{n}\right)$-Laplacian, J. Math. Anal. Appl. 369 (2010), 76-81.

[9] D. ÇAKMAK, On Lyapunov-type inequality for a class of nonlinear systems, Math. Inequal. Appl. 16 (2013), 101-108.

[10] R. S. DAhiYA, B. Singh, A Liapunov inequality and nonoscillation theorem for a second order nonlinear differential-difference equations, J. Math. Phys. Sci. 7 (1973), 163-170.

[11] O. Dosly, P. Rehak, Half-Linear Differential Equations, Mathematics Studies 202, North-Holland 2005.

[12] A. Elbert, A half-linear second order differential equation, Colloq. Math. Soc. Janos Bolyai 30 (1979), 158-180.

[13] S. B. Eliason, A Lyapunov inequality, J. Math. Anal. Appl. 32 (1970), 461-466.

[14] S. B. EliAson, A Lyapunov inequality for a certain nonlinear differential equation, J. London Math. Soc. 2 (1970), 461-466.

[15] S. B. Elias ON, Lyapunov type inequalities for certain second order functional differential equations, SIAM J. Appl. Math. 27, 1 (1974), 180-199.

[16] G. Guseinov, B. KaymaKÇAlan, Lyapunov inequalities for discrete linear Hamiltonian system, Comput. Math. Appl. 45 (2003), 1399-1416. 
[17] P. Hartman, Ordinary Differential Equations, Wiley, New York, 1964 an Birkhäuser, Boston 1982.

[18] L. JiAnG, Z. ZHOU, Lyapunov inequality for linear Hamiltonian systems on time scales, J. Math. Anal. Appl. 310 (2005), 579-593.

[19] M. K. Kwong, On Lyapunov's inequality for disfocality, J. Math. Anal. Appl. 83 (1981), 486-494.

[20] C. Lee, C. Yeh, C. Hong, R. P. Agarwal, Lyapunov and Wirtinger inequalities, Appl. Math. Letters 17 (2004), 847-853.

[21] P. L. NAPOLI, J. P. PinAsCo, Estimates for eigenvalues of quasilinear elliptic systems, J. Differential Equations 227 (2006), 102-115.

[22] B. G. PAChPATTE, On Lyapunov-type inequalities for certain higher order differential equations, J. Math. Anal. Appl. 195 (1995), 527-536.

[23] B. G. PAChPATte, Lyapunov type integral inequalities for certain differential equations, Georgian Math. J. 4, 2 (1997), 139-148.

[24] B. G. PACHPATtE, Inequalities related to the zeros of solutions of certain second order differential equations, Facta Universitatis Ser. Math. Inform. 16 (2001), 35-44.

[25] S. PANIGRAHI, Lyapunov-type integral inequalities for certain higher order differential equations, Electron. J. Differential Equations Vol. 2009, 28 (2009), 1-14.

[26] N. PARhi, S. PANIGRAhi, On Liapunov-type inequality for third-order differential equations, J. Math. Anal. Appl. 233, 2 (1999), 445-460.

[27] N. Parhi, S. PANigrahi, Liapunov-type inequality for higher order differential equations, Math. Slovaca 52, 1 (2002), 31-46.

[28] J. P. PinASCO, Lower bounds for eigenvalues of the one-dimensional p-Laplacian, Abstr. Appl. Anal. 2004, 2 (2004), 147-153.

[29] J. P. PinAsco, Comparison of eigenvalues for the p-Laplacian with integral inequalities, Appl. Math. Comput. 182 (2006), 1399-1404.

[30] T. W. REID, A matrix Lyapunov inequality, J. Math. Anal. Appl. 32 (1970), 424-434.

[31] I. SIM, Y. LEE, Lyapunov inequalities for one-dimensional p-Laplacian problems with a singular weight function, J. Inequal. Appl. 2010, Art. ID 865096, 9 pp.

[32] X. H. TANG, X. HE, Lower bounds for generalized eigenvalues of the quasilinear systems, J. Math. Anal. Appl. 385 (2012), 72-85.

[33] A. TIRYAKI, Recent developments of Lyapunov-type inequalities, Adv. Dyn. Syst. Appl. 5 (2010), 231-248.

[34] A. TIRYAKI, M. ÜNAL, D. ÇAKMAK, Lyapunov-type inequalities for nonlinear systems, J. Math. Anal. Appl. 332 (2007), 497-511.

[35] A. TIRYAKI, D. ÇAKMAK, M. F. AKTAŞ, Lyapunov-type inequalities for a certain class of nonlinear systems, Comput. Math. Appl. 64 (2012), 1804-1811.

[36] M. ÜNAL, D. ÇAKMAK, A. TIRYAKI, A discrete analogue of Lyapunov-type inequalities for nonlinear systems, Comput. Math. Appl. 55 (2008), 2631-2642.

[37] M. ÜNAL, D. ÇAKMAK, Lyapunov-type inequalities for certain nonlinear systems on time scales, Turkish J. Math. 32 (2008), 255-275.

[38] X. YAnG, On Liapunov-type inequality for certain higher-order differential equations, Appl. Math. Comput. 134 (2003), 307-317.

[39] X. YANG, K. Lo, Lyapunov-type inequality for a class of even-order differential equations, Appl. Math. Comput. 215 (2010), 3884-3890.

[40] X. YANG, Y. KIM, K. Lo, Lyapunov-type inequality for a class of odd-order differential equations, J. Comput. Appl. Math. 234 (2010), 2962-2968.

[41] X. YANG, Y. KIM, K. Lo, Lyapunov-type inequality for a class of quasilinear systems, Math. Comput. Modelling 53 (2011), 1162-1166.

[42] X. WANG, Lyapunov type inequalities for second-order half-linear differential equations, J. Math. Anal. Appl. 382 (2011), 792-801.

[43] G. A. Afrouzi, S. Heidarkhani, Existence of three solutions for a class of Dirichlet quasilinear elliptic systems involving the $\left(p_{1}, p_{2}, \ldots, p_{n}\right)$-Laplacian, Nonlinear Anal. 70 (2009), 135-143. 